\title{
Ride-sharing platforms from drivers' perspective: Evidence from Uber and Lyft drivers
}

\author{
Sina Shokoohyar ${ }^{\mathrm{a}^{*}}$
}

\begin{tabular}{l}
${ }^{a}$ Saint Joseph's University, United Stat \\
\hline C H R O N I C L E \\
\hline Article history: \\
Received: June 2, 2018 \\
Received in revised format: Sep- \\
tember 20, 2018 \\
Accepted: October 10, 2018 \\
Available online: \\
October 10, 2018 \\
\hline Keywords: \\
Ride-sharing Platform \\
Supply Side Management \\
Job Satisfaction \\
Uber and Lyft \\
\hline
\end{tabular}

\begin{abstract}
A B S T R A C T
Uber and its main competitor Lyft are competing aggressively to attract more drivers and in turn limit the access of their competitors in the drivers' supply base. To control the supply side, it is important to analyze the ride-sharing platforms from drivers' perspective. Using Uber and Lyft drivers' online reviews, it is shown that drivers are slightly rating Uber higher than Lyft. Additionally, comparing Uber and Lyft rating trend over time, the analysis shows that they are closely competing to attract more drivers. On the aggregate level, drivers count job flexibility, work/life balance, and meeting new people as the main advantages of working in the ride-sharing platforms. On the other hand, the results show that drivers suffer from insufficient compensation of their operating costs, poor job security, experiencing bad riders' behavior, and poor customer service.
\end{abstract}

C 2018 by the authors; licensee Growing Science, Canada.

\section{Introduction}

Ride-sharing platforms (e.g., Uber, Lyft, Grab, GoJek, EasyTaxi, Hitch-a-ride, Didi Chuxing) have grown significantly during recent years (Dogtiev, 2017; Kokalitcheva, 2016). In 2017 Uber, car rentals, Lyft and taxi shared 54\%, 28\%, 11\% and 7\% of United States ride-hailing market, respectively (Certify, 2017; Newcomer, 2017). Excluding rental cars, Uber and Lyft covered 91\% of the market. On-demand ride-sharing platforms enable people to share travel related expenses by sharing rides. This platform is simply based on the mobile apps. Passengers can enter pickup location and destination in the app and the app will provide them with the list of possible services and their fares. Then the passenger can select a service and request a ride. The app then matches the rider with a driver-partner. To engineer a perfectly efficient system, a balance between rider demand and driver supply are needed to offer the lowest cost ride to passengers and the company (Scheiber, 2017). In this platform, the labor supply is not controlled by the company but instead by the numerous independent contractors (Redfearn III, 2016). The large size of the supply base decreases drivers' bargaining power and in turn makes it very easy for platforms to decrease the trip fare. In 2014, unhappy Uber drivers protest against Uber. They demonstrate against several issues including low fares, tipping policy, rating system, and driver safety (Kosoff, 2014).

* Corresponding author.

E-mail address: sshokooh@sju.edu (S. Shokoohyar)

(C) 2018 by the authors; licensee Growing Science, Canada. doi: $10.5267 /$ j.ijdns.2018.10.001 
In the ride-sharing platform it is essential for the business managers to be able to control the supply side (drivers) to match the growing demand. Uber and its main competitor Lyft are offering several different incentive contracts to their driver partners and are issuing new policies to regulate and control the supply side. For instance, to encourage the driver supply, on top of dynamically pricing the trips fare, Uber offers rewards to its drivers if they achieve a pre-specified number of trips per week (e.g. drivers receive \$235 when they complete 100 trips per week). Therefore, Uber can incentivize its desirable behavior of its drivers by manipulating the per week reward. Ride-sharing companies also appear to be competing for drivers and their promotion and incentives are getting closer to each other over time (Rideshare Central, 2017). Motivated by these observations, this study investigates the following research questions regarding ride-sharing platforms. First, are there any differences between Uber and Lyft from drivers' perspective? Second, overall what are the ups and downs of working as a driver partner in the ride-sharing platform? What do attract drivers in working as an independent contractor in the ride-sharing platform?

To address these research questions, 7183 drivers' online reviews (5911 Uber and 1272 Lyft reviews) are analyzed. The results show that drivers rate working condition at Uber slightly higher than Lyft. Additionally, Uber's and Lyft overall rating are getting closer to each other over time, indicating that they are competing closely to attract more drivers. On the aggregate level, the result shows that job flexibility, the balance between life and work, and the ability of meeting new people are the main factors that attract drivers. On the other hand, drivers mostly count insufficient compensation of the operating costs, poor job security, experiencing bad behavior of the riders, and poor customer service as the cons of working in the ride-sharing platforms.

In what follows, the literature is reviewed in Section 2 before collected data and applied methodology being described in Section 3. Section 4 then presents obtained result. Finally, the results are discussed and concluded in Section 5.

\section{Literature Review}

Studies in the area of ride-sharing platforms are widespread, and different aspect of ride-sharing platform have been studied. There exists a vast literature on matching supply and demand through dynamic pricing, known as "surge" pricing. Guda and Subramanian (2018) investigated how surge pricing can be used as a tool to communicate the forecasted demand to drivers and in turn increase supply of drivers. Jiao (2018) studied the impact of special events (i.e., $4^{\text {th }}$ of July in their study) in Austin, TX on the magnitude of Uber surge price. Cohen and Zhang (2017) analyzed the price competition between ride-sharing platforms, and analyzed introducing a new joint service by them. Jacob and Roet-Green (2017) derived the optimal pricing of ride-sharing platform services (i.e., solo and pool rides). Chen and Sheldon (2016) studied the impact of dynamic pricing of trips on drivers' behavior in the ride-sharing platform. Brodeur and Nield (2016) studied the impact of rainy weather condition on number of provided Uber rides in New York city. They showed that as increase in the surge multiplier during the rainy weather condition increases the supply. Bimpikis et al. (2016) investigated the impact of demand pattern over the network on the equilibrium price. Hall et al. (2015) provided evidence on how the Uber surge pricing algorithm equilibrates the supply and demand. Chen et al. (2015) analyzed the Uber surge pricing algorithm and explored approaches to predict it and ways to avoid it. Banerjee et al. (2015) used a queueing-theoretic approach to derive the optimal dynamic pricing in the ride-sharing platforms. For a literature review on dynamic optimization of ride-sharing platforms, the readers are referred to (Agatz, Erera, Savelsbergh, \& Wang, 2012).

Some research papers studied the environmental and socio-economic aspect of ride-sharing platforms. Studies have shown that car-sharing platforms (such as Zipcar and car2go) significantly reduces greenhouse gas emissions (Firnkorn \& Müller, 2011; Martin \& Shaheen, 2011). Wang and Mu (2018) studied impact of race, wealth and population density on Uber accessibility. They showed that Uber accessibility is not correlated with wealth and race and on the other hand it is correlated with road network density, and population density. This paper is concerned with the supply side of the ride-sharing platforms. Oei and Ring (2017) studied Uber and Lyft drivers' tax issues and challenges. They showed that drivers 
display accurate understandings of tax filing. On the other hand, their approaches to expenses and deductions are less accurate. Chen et al. (2017) studied the value of working time flexibility for Uber drivers. The showed that real-time flexibility is more beneficial for Uber drivers in terms of earning compared to the less flexible arrangements. Some studies argued the differences between regular employees and independent contractors (drivers) in ride-sharing platforms from the law perspective (Davidov, 2016; Rassman, 2014; Redfearn III, 2016; Woo \& Bales, 2017). Malin and Chandler (2017) investigated the impact of on-demand employment on drivers. They interviewed with 18 Pittsburg-based Uber and Lyft drivers. They observe that flexibility and sociability are the main advantages of the working in the ridesharing platform from the drivers' perspective. On the other hand, they showed that surge pricing force long working hours through weekends. Additionally, they observed that drivers suffer from some bad behavior of riders. Cramer and Krueger (2016) studied capacity utilization of Uber drivers and compared it with that of taxi drivers. They showed that Uber $\mathrm{x}$ drivers have a significantly higher capacity utilization compared to taxi drivers. Stiglic et al. (2016) investigated the impact of flexibility on the performance of a driver, rider and ride-sharing system. They showed that the expected matching rate between drivers and riders increases significantly in flexibility. Hall and Krueger (2015) studied labor market of Uber's driver-partners. They showed that Uber drivers are attracted to platform because of flexibility, and compensation. Rogers (2015) studied the social costs of using Uber. He discussed several concerns about Uber services including safety, privacy, discrimination, and labor standards. Feeney and companies Uber (2015) investigated the safety of drivers and riders in ride-sharing platforms.

The aforementioned studies differ from this paper in two main points. First, in terms of the research methodology and the available data. Unlike them this research uses a much larger dataset that allows to dig deeper and provide more insights into drivers' perspective of the ride-sharing platform. Through sentiment analysis, most important features that leads to drivers' job satisfaction as well as job dissatisfaction are classified. This paper studies both ups and downs of working as a driver in the ride-sharing platforms. Second, this paper analyzes and compares drivers' rating of Uber and Lyft in five rating categories as well as their overall rating over time.

\section{Data and Methodology}

In this study, drivers who worked as a driver partner in Uber and Lyft and reviewed the working conditions on indeed.com are considered. Indeed.com is a search engine for job listing that is lunched in November 2005. It provides variety of services like job search, recommended job, job trends, résumé upload, salary search, job competition index, and company reviews. The following attributes are extracted for each online review: employer, job location, review date, review title, review, pros, cons, job culture rating, management rating, job security/advancement rating, compensation/benefits rating, job work/life balance rating, and overall rating. These attributes are presented in Table 1.

In total, 7183 reviews (5911 Uber and 1272 Lyft reviews) are acquired. In analysis, instead of the rating with 5 stars scaling, the ratings are converted to numerical rating scale from 100.

To address the first research question (i.e., are there any differences between Uber and Lyft in terms of drivers' job satisfaction?) Uber and Lyft are compared in terms of drivers' job satisfaction in all of the five rating categories using two-sided t-tests. Additionally, Uber and Lyft ratings trend are analyzed and compared using linear regression model. The results are presented in Section 4.1.

To respond to the second research question (i.e., overall what are the ups and downs of working as a driver partner in the ride-sharing platform? What do attract drivers in working as an independent contractor in the ride-sharing platform?) pros and cons as well as the review content are analyzed. Sentiment analysis techniques is used to organize the message conveyed through the review content. Sentiment analysis is widely used to analyze online reviews (Pang \& Lee, 2005; Pang, Lee, \& others, 2008; Prabowo \& Thelwall, 2009; Ye, Zhang, \& Law, 2009; Yu, Liu, Huang, \& An, 2012). For a literature review on opinion mining and sentiment analysis approaches, the readers are referred to (Liu \& Zhang, 2012; Vinodhini \& Chandrasekaran, 2012). Naive Bayes classifier is a popular and simple machine learning 
technique for text classification, and it performs well in many domains (Domingos \& Pazzani, 1997). This method is applied to analyze the content of the online reviews and the results are presented in Section

4.2 .

Table 1

Attributes Extracted from Online Reviews

\begin{tabular}{ll}
\hline Attribute & Description \\
\hline Employer & Uber or Lyft \\
Job Location & Location of the job \\
Review Date & Date the review is created \\
Review Title & Title of posted review \\
Review & Content of the review \\
Pros & Working advantages \\
Cons & Working disadvantages \\
Job Culture Rating & Rating by reviewer $(0$ to 5 stars $)$ \\
Management Rating & Rating by reviewer $(0$ to 5 stars $)$ \\
Job Security/Advancement Rating & Rating by reviewer $(0$ to 5 stars $)$ \\
Compensation/Benefits Rating & Rating by reviewer $(0$ to 5 stars $)$ \\
Job Work/Life Balance Rating & Rating by reviewer $(0$ to 5 stars $)$ \\
Overall Rating & Generated average rating $(0$ to 5 stars $)$ \\
\hline
\end{tabular}

\section{Results}

In what follows, Uber and Lyft are analyzed and compared based on drivers' reviews in Section 4.1. Next in Section

4.2, aggregate drivers' reviews are explored to identify important factors in derivers job satisfaction.

\subsection{Uber or Lyft?}

Fig. 1 shows drivers' rating of Uber (black bars) and Lyft (purple bars) in 5 rating categories as well as the overall rating separately. Table 2 summarizes descriptive statistics of rating categories as well as the statistical test results. Rating between Uber and Lyft are formally compare using a one-sided t-test, and the alternative hypothesis as implied by column six of Table 2. The unit of analysis is a deriver's review. Under 5\% significant level, the results indicate that Uber receives higher rating in Job Security/Advancement and Compensation/Benefit compared to the Lyft. On the other hand, Lyft has a higher rating in Job culture category. Based on the statistical tests, the statement that Uber and Lyft have a same rating in both Job Culture and Management categories cannot be rejected. Overall, the result shows that Uber achieves a higher rating compared to Lyft.

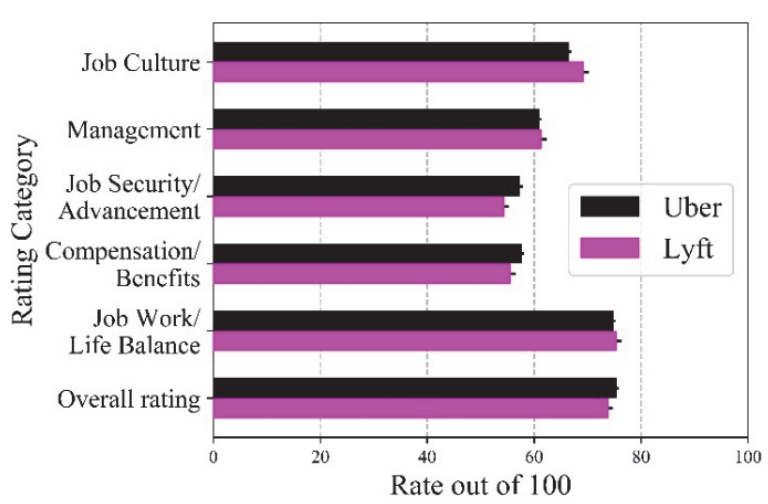

Fig. 1. Drivers' Rating of Uber and Lyft

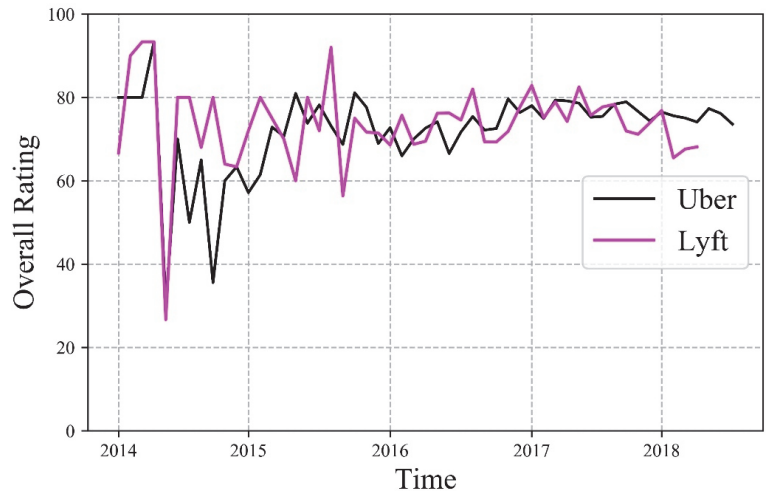

Fig. 2. Uber and Lyft Overall Rating Over Time

Fig. 2 shows the overall rating of Uber (black line) and Lyft (purple line) over time. The figure presents a sense of how their overall rating evolves over time and compare to each other. The figure provides two main observations. First, overall ratings of Uber and Lyft are in vicinity of each other. This observation 
indicates that Uber and Lyft tend to compete in achieving a better drivers' job satisfaction and in turn increase their share of drivers' base. Second, overall rating is improving over time.

Table 2

Summary of Descriptive Statistics and Statistical Tests

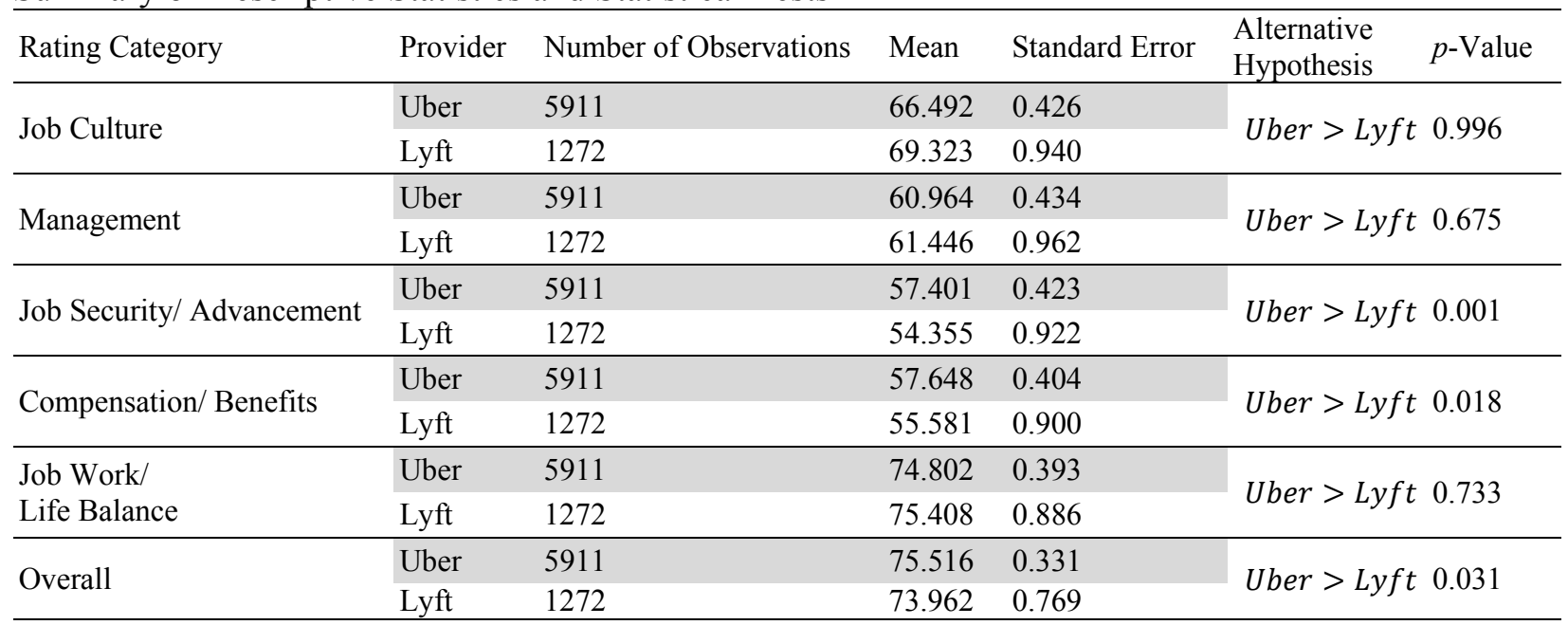

To test these observations, OLS regression is run where the dependent variable is the Uber overall rating and the independent variables as presented in the first row of Table 3. Note that Model (2) results in a higher adjusted R-squared. The positive and significant coefficient of Lyft Overall Rating shows that Uber and Lyft compete closely to improve their overall rating. The positive and significant coefficient of the Time shows that overall Rating is increasing over time. Note that in each cell of Table 3, the first, second and third row presents the coefficient estimation, standard error (in parenthesis), and $p$-Value (in italic format), respectively.

Table 3

Summary of the OLS Regression

\begin{tabular}{lllll}
\hline Independent Variables & Lyft's Overall Rating & Time & Constant & Adj. $R^{2}$ \\
\hline \multirow{3}{*}{ Model (1) } & 0.28 & & 53.88 & \\
& $(0.092)$ & -- & $(6.761)$ & 0.173 \\
& 0.004 & & 0.000 & \\
Model (2) & 0.25 & 0.11 & 53.84 & \\
& $(0.088)$ & $(0.048)$ & $(6.425)$ & 0.253 \\
\hline
\end{tabular}

\subsection{Aggregate Reviews (Sentiment Analysis)}

In this Section, Uber and Lyft reviews are combined to investigate main attributes leading in drivers' job satisfaction in the ride-sharing platforms. Fig. 3 represents drivers rating of working conditions in all the five rating categories. Note that the rating categories are organized in the ascending order from top to bottom. This figure provides two main observations. First, the main advantage of working in the ridesharing platform as a driver partner are the Job Work/Life Balance and Job Culture. Second, Job Security/Advancement and Compensation/Benefits receive very low rating. These observations indicate that although working as a driver provides very flexible schedule and good balance between work and life, it does not compensate drivers' costs of operation and has a very poor job security. 


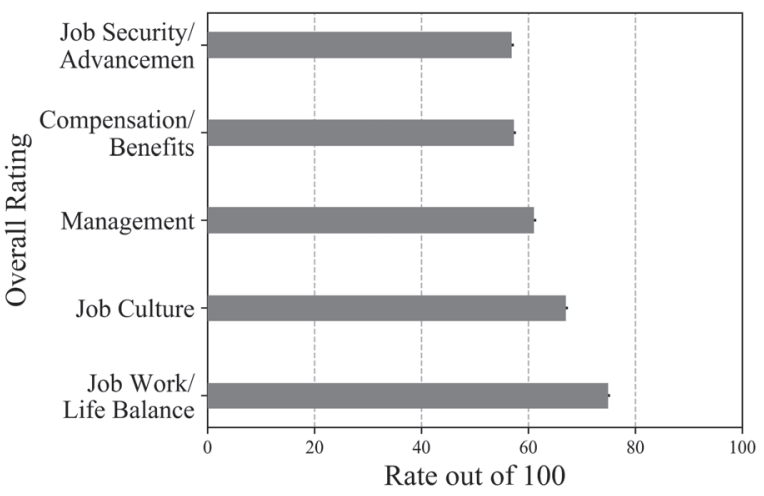

Fig. 3. Aggregated Overall Rating

Fig. 4 (the left figure with the white background) shows the most commonly used word in the pros of working as a driver in the ride-sharing platforms. The figure reveals two main observations. Pros are mostly associated with the working time flexibility and the experience of meeting new people (riders). One of the main factors that distinguish the ride-sharing platform with the more conventional jobs (e.g., taxi driver, bus driver, ...) is the flexibility of the working time. In the ride-sharing platforms, drivers are not restricted by a fixed working schedule. Drivers are free on deciding when to work. Drivers in the ride-sharing platform can also select the location (i.e., origin and destination of the trip) which provides them with even higher level of flexibility. Additionally, the experience of meeting new people (riders, customers) makes the job more fun for the drivers.

Fig. 5 (the right figure with the black background) represents the most commonly used word in the cons of working as a driver in the ride-sharing platforms. This figure shows that drivers are mostly concerned about the compensation of the operating costs (vehicle costs and expenses (wear and tear), gas, car mileage) and whether it worth the time that they spend working as a driver partner of Uber or Lyft. Drivers are also suffering from poor and awful customer service provided. Additionally, having a bad experience with passenger (customer, rider) are mentioned as cons of working in the ride-sharing platform.

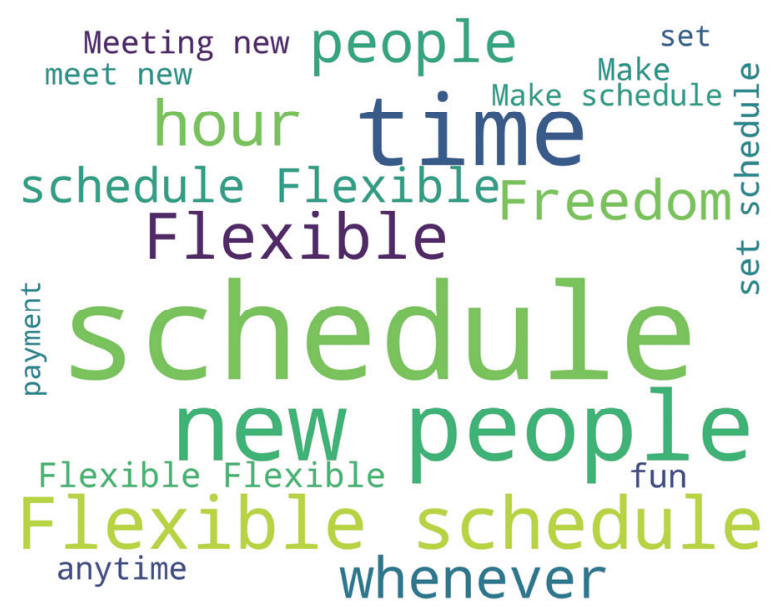

Fig. 4. Most Commonly Used Words in Pros

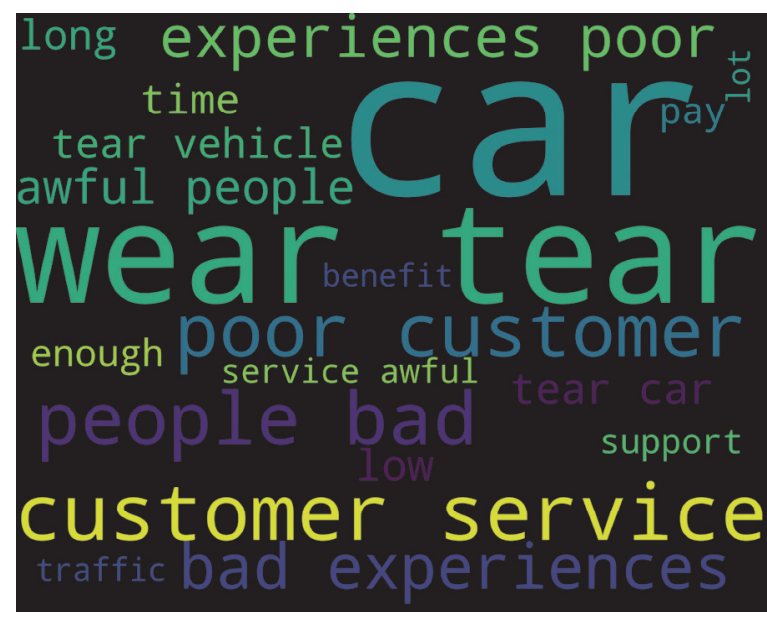

Fig. 5. Most Commonly Used Words in Cons

Next, aggregated drivers' reviews (i.e., aggregate reviews of both Uber and Lyft) are analyzed to identify the main deriving factors of positive and negative reviews. Reviews are categorized with overall rating below $40 \%$ (up to 2 stars), between $40 \%$ and $60 \%$ ( 3 stars) and above $60 \%$ (4 and 5 stars) as negative, neutral, and positive, respectively.

Table 4 presents the correlation matrix among every two rating factors. Under 1\% significant level, the statement that the rating factors are not correlated is rejected. As all rating categories are highly correlated, reviews' sentiment is categorized based on the overall rating. 
Table 4

Correlation Among Rating Categories

\begin{tabular}{lccccc}
\hline Rating Category & Job Culture & Management & $\begin{array}{c}\text { Job Security/ } \\
\text { Advancement }\end{array}$ & $\begin{array}{c}\text { Compensation/ } \\
\text { Benefits }\end{array}$ & $\begin{array}{c}\text { Job Work/ } \\
\text { Life Balance }\end{array}$ \\
\hline Job Culture & NA & 0.79 & 0.72 & 0.70 & 0.69 \\
Management & 0.79 & NA & 0.76 & 0.71 & 0.62 \\
Job Security/Advancement & 0.72 & 0.76 & NA & 0.77 & 0.60 \\
Compensation/Benefits & 0.70 & 0.71 & 0.77 & NA & 0.62 \\
Job Work/Life Balance & 0.69 & 0.62 & 0.60 & 0.62 & NA \\
\hline
\end{tabular}

The Naive Bayes Classifier is applied to identify the important features of drivers' review text that leads to a positive or negative review. The result of the classification is presented in Table 5 along with the enumeration of the feature sets in the last column. Note that the Pos and Neg stands for Positive and Negative, respectively. In the 10 most important features Meeting, Great and Environment are features that are associated with positive reviews. On the other hand, Desperate, Fee, Policy, Lie, False, Charging, and Net are features that are mostly associated with the negative reviews. $80 \%$ of the reviews are included to train the classifier and the remaining for testing it. The accuracy of the classifier is $80 \%$.

\section{Table 5}

10 Most Informative Features Given by the Naive Bayes Classifier

\begin{tabular}{llll}
\hline$\#$ & Feature & & Ratio \\
\hline 1 & Desperate & Neg: Pos & \\
2 & Fee & Neg: Pos & $42.0: 1.0$ \\
3 & Policy & Neg: Pos & $31.0: 1.0$ \\
4 & Lie & Neg: Pos & $27.0: 1.0$ \\
5 & False & Neg : Pos & $23.9: 1.0$ \\
6 & Meeting & Pos $:$ Neg & $20.1: 1.0$ \\
7 & Charging & Neg : Pos & $19.8: 1.0$ \\
8 & Great & Pos : Neg & $18.3: 1.0$ \\
9 & Net & Neg : Pos & $17.8: 1.0$ \\
10 & Environment & Pos : Neg & $17.5: 1.0$ \\
\hline
\end{tabular}

Classifier Accuracy: 80\%

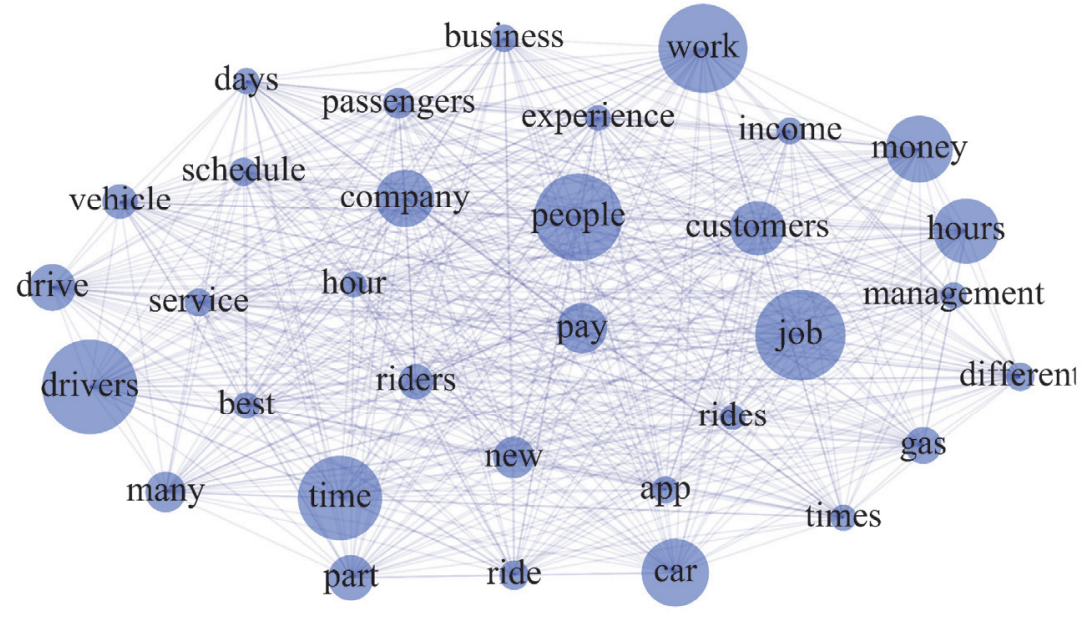

Fig. 6. Most Commonly Used Words Drivers’ Review 
Next, centering resonance analysis (CRA) (Corman et al., 2002) is applied to identify important words in drivers' reviews and link these into a network. Influential words are those creating noun phrases, that are potential centers in the reviews. Betweenness centrality is used in estimating the influence of each word in CRA. Fig. 6 represents the most important features extracted based on CRA. The result is in line with aforementioned analysis of pros and cons of driving in the ride-sharing platforms.

\section{Conclusion}

This paper studies drivers' concerns in working in the ride-sharing platforms like Uber and Lyft. 7183 driver's reviews along with the rating score in five categories (i.e., Job Culture, Management, Job Security/Advancement, Compensation/Benefits, and Job Work/Life Balance) are collected from Indeed.com. Drivers who worked for Uber and Lyft and express their experiences of working as a driver in Uber and Lyft on Indeed.com.

These reviews are first separately analyzed to compared Uber and Lyft in terms of drivers' job satisfaction. The statistical results in this section provides two main findings. First, drivers' rate Uber slightly higher compared to Lyft. This result indicates that Uber's policies are more successful in attracting drivers. Second, both Uber and Lyft are closely competing, and their overall ratings are increasing over time.

Next, reviews of both Uber and Lyft drivers are aggregated and analyzed to identify the ups and downs of working in the ride-sharing platforms. The results show that the main advantage of working as a driver in the ride-sharing platforms are the job flexibility, the balance between life and work, and the ability of meeting new people. On the other hand, drivers suffer from insufficient compensation of the operating costs, poor job security, experiencing bad behavior of the riders, and poor customer service.

This study provides two main managerial implications. First, the results show that drivers enjoy meeting new and nice riders, and on the other hand suffer from bad riders' behavior. This result indicates that the ride-sharing platform can benefits from regulating driver-rider relationship to improve drivers' job satisfaction. To encourage better rider behavior, both Uber and Lyft have established rider rating system on their apps which allows drivers to rate riders behavior (Lyft, 2018a; Truong \& Trivedi, 2017). Second, drivers are mainly concerned about the operational costs and lack of job security. Providing more supportive incentive contract to compensate drivers' operational costs can improve drivers job satisfaction. Uber and Lyft offer rental options to driver such that they do not need to be worried about the operational cost as well as insurance coverage (Lyft, 2018b; Uber, 2018). The managerial insight provided in this paper will help ride-sharing platform to manage and improve the supply side (drivers base). To be able to meet demand, improving supply side is essential as ride-sharing platforms share of the transportation market is growing rapidly (Certify, 2017; Dogtiev, 2017).

\section{References}

Agatz, N., Erera, A., Savelsbergh, M., \& Wang, X. (2012). Optimization for dynamic ride-sharing: A review. European Journal of Operational Research, 223(2), 295-303.

Banerjee, S., Riquelme, C., \& Johari, R. (2015). Pricing in ride-share platforms: A queueing-theoretic approach.

Bimpikis, K., Candogan, O., \& Daniela, S. (2016). Spatial pricing in ride-sharing networks.

Brodeur, A., \& Nield, K. (2016). Has Uber Made It Easier to Get a Ride in the Rain?

Certify. (2017). Uber Declines, Lyft Picks Up in the Certify SpendSmart ${ }^{\mathrm{TM}}$ Report for Q3 2017. Retrieved from https://www.certify.com/2017-10-24-Uber-Declines-Lyft-Picks-Up-in-the-CertifySpendSmart-Report-for-Q3-2017

Chen, L., Mislove, A., \& Wilson, C. (2015). Peeking beneath the hood of uber. In Proceedings of the 2015 Internet Measurement Conference (pp. 495-508).

Chen, M. K., Chevalier, J. A., Rossi, P. E., \& Oehlsen, E. (2017). The value of flexible work: Evidence from uber drivers.

Chen, M. K., \& Sheldon, M. (2016). Dynamic Pricing in a Labor Market: Surge Pricing and Flexible 
Work on the Uber Platform. In $E C$ (p. 455).

Cohen, M. C., \& Zhang, R. P. (2017). Coopetition and profit sharing for ride-sharing platforms.

Cramer, J., \& Krueger, A. B. (2016). Disruptive change in the taxi business: The case of Uber. American Economic Review, 106(5), 177-182.

Davidov, G. (2016). The status of Uber drivers: A purposive approach.

Dogtiev, A. (2017). Uber Revenue and Usage Statistics. Retrieved from http://www.businessofapps.com/data/uber-statistics/

Domingos, P., \& Pazzani, M. (1997). On the optimality of the simple Bayesian classifier under zero-one loss. Machine Learning, 29(2-3), 103-130.

Feeney, M., \& companies Uber, R. (2015). Is Ridesharing Safe?

Firnkorn, J., \& Müller, M. (2011). What will be the environmental effects of new free-floating carsharing systems? The case of car2go in Ulm. Ecological Economics, 70(8), 1519-1528.

Guda, H., \& Subramanian, U. (2018). Your Uber Is Arriving: Managing On-Demand Workers through Surge Pricing, Forecast Communication and Worker Incentives.

Hall, J., Kendrick, C., \& Nosko, C. (2015). The effects of Uber's surge pricing: A case study. The University of Chicago Booth School of Business.

Hall, J. V, \& Krueger, A. B. (2015). An analysis of the labor market for Uber's driver-partners in the United States. ILR Review, 0019793917717222.

Jacob, J., \& Roet-Green, R. (2017). Ride Solo or Pool: The Impact of Sharing on Optimal Pricing of Ride-Sharing Services.

Jiao, J. (2018). Investigating Uber price surges during a special event in Austin, TX. Research in Transportation Business \& Management.

Kokalitcheva, K. (2016). Uber now has 40 million monthly riders worldwide. Fortune Magazine.

Kosoff, M. (2014). Uber Drivers Across The Country Are Protesting Today: Here's Why. Business Insider, 22.

Liu, B., \& Zhang, L. (2012). A survey of opinion mining and sentiment analysis. In Mining text data (pp. 415-463). Springer.

Lyft. (2018a). Driver and passenger ratings. Retrieved from https://help.lyft.com/hc/enus/articles/115013079948-Driver-and-passenger-ratings\#passengerrating

Lyft. (2018b). Express Drive Rental Car Program. Retrieved from https://help.lyft.com/hc/enus/articles/115013080108-Express-Drive-Rental-Car-Program

Malin, B. J., \& Chandler, C. (2017). Free to work anxiously: Splintering precarity among drivers for Uber and Lyft. Communication, Culture \& Critique, 10(2), 382-400.

Martin, E. W., \& Shaheen, S. A. (2011). Greenhouse gas emission impacts of carsharing in North America. IEEE Transactions on Intelligent Transportation Systems, 12(4), 1074-1086.

Newcomer, E. (2017). Lyft Gains on Uber in U.S. Business Travel, Doubling Share. Retrieved from https://www.bloomberg.com/news/articles/2018-04-30/lyft-gains-on-uber-in-u-s-business-traveldoubling-share

Oei, S.-Y., \& Ring, D. M. (2017). The Tax Lives of Uber Drivers: Evidence from Internet Discussion Forums. Colum. J. Tax L., 8, 56.

Pang, B., \& Lee, L. (2005). Seeing stars: Exploiting class relationships for sentiment categorization with respect to rating scales. In Proceedings of the 43rd annual meeting on association for computational linguistics (pp. 115-124).

Pang, B., Lee, L., \& others. (2008). Opinion mining and sentiment analysis. Foundations and Trends ${ }^{\circledR}$ in Information Retrieval, 2(1--2), 1-135.

Prabowo, R., \& Thelwall, M. (2009). Sentiment analysis: A combined approach. Journal of Informetrics, $3(2), 143-157$.

Rassman, C. L. (2014). Regulating rideshare without stifling innovation: Examining the drivers, the insurance gap, and why Pennsylvania should get on board. Pitt. J. Tech. L. \& Pol'y, 15, 81.

Redfearn III, R. L. (2016). Sharing economy misclassification: Employees and independent contractors in transportation network companies. Berkeley Tech. LJ, 31, 1023.

Rideshare Central. (2017). Lyft Driver Promos vs. Uber Driver Promos. Retrieved from 
https://ridesharecentral.com/lyft-driver-promos-vs-uber-driver-promos

Rogers, B. (2015). The social costs of Uber. U. Chi. L. Rev. Dialogue, 82, 85.

Scheiber, N. (2017). How Uber uses psychological tricks to push its drivers' buttons. The New York Times, 2.

Stiglic, M., Agatz, N., Savelsbergh, M., \& Gradisar, M. (2016). Making dynamic ride-sharing work: The impact of driver and rider flexibility. Transportation Research Part E: Logistics and Transportation Review, 91, 190-207.

Truong, M., \& Trivedi, R. (2017). Updates to the Rating System. Retrieved from https://www.uber.com/newsroom/ratingsupdate-2/

Uber. (2018). Car Offers, Wheels by the Week. Retrieved from https://www.uber.com/drive/vehiclesolutions/

Vinodhini, G., \& Chandrasekaran, R. M. (2012). Sentiment analysis and opinion mining: a survey. International Journal, 2(6), 282-292.

Wang, M., \& Mu, L. (2018). Spatial disparities of Uber accessibility: An exploratory analysis in Atlanta, USA. Computers, Environment and Urban Systems, 67, 169-175.

Woo, C., \& Bales, R. A. (2017). The Uber Million Dollar Question: Are Uber Drivers Employees or Independent Contractors?

Ye, Q., Zhang, Z., \& Law, R. (2009). Sentiment classification of online reviews to travel destinations by supervised machine learning approaches. Expert Systems with Applications, 36(3), 6527-6535.

Yu, X., Liu, Y., Huang, X., \& An, A. (2012). Mining online reviews for predicting sales performance: A case study in the movie domain. IEEE Transactions on Knowledge and Data Engineering, 24(4), 720734.

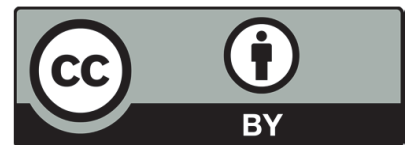

(C) 2018 by the authors; licensee Growing Science, Canada. This is an open access article distributed under the terms and conditions of the Creative Commons Attribution (CCBY) license (http://creativecommons.org/licenses/by/4.0/). 